\title{
Exotic Axion Cosmology in Theories with Phase Transitions below the QCD Scale
}

\author{
David B. Kaplan* and Kathryn M. Zurek ${ }^{\dagger}$ \\ Institute for Nuclear Theory, University of Washington, Seattle, Washington 98195, USA
}

(Received 28 July 2005; published 30 January 2006)

\begin{abstract}
We show that axion phenomenology may be significantly different than conventionally assumed in theories which exhibit late phase transitions (below the QCD scale). In such theories, one can find multiple pseudoscalars with axionlike couplings to matter, including a string scale axion, whose decay constant far exceeds the conventional cosmological bound. Such theories have several dark matter candidates.
\end{abstract}

PACS numbers: $98.80 . \mathrm{Cq}, 14.80 . \mathrm{Mz}, 95.35 .+\mathrm{d}$

Introduction. - The smallness of the experimentally determined upper bound on the strong $C P$ violating parameter, $\bar{\theta} \lesssim 10^{-9}$, is an outstanding puzzle of the standard model. One can either assume $C P$ to be an exact symmetry spontaneously broken in such a way as to ensure that $\bar{\theta}$ is naturally small, as in the Nelson-Barr mechanism [1,2], or one can introduce a $U(1)$ symmetry, known as the PecceiQuinn (PQ) symmetry $[3,4]$ to allow $\bar{\theta}$ to dynamically relax to zero. (Spontaneous breaking of parity has also been suggested $[5,6]$.) An attractive feature of the PQ mechanism is that it divorces the strong $C P$ problem from flavor physics - the masses and mixings of the quarks - whose origin remains a mystery. The PQ mechanism entails a global $U(1)$ symmetry which is exact up to a QCD (and possibly electromagnetic) anomaly. The symmetry breaks spontaneously at a scale $f$, giving rise to a pseudoscalar Goldstone boson, the axion, which couples to matter via the interaction $(a / f) G \tilde{G}[7-12]$. Here $a$ is the axion, $f$ is its decay constant, $G_{\mu \nu}$ is the gluon field strength, and the ratio $(a / f)$ should be thought of as an angle. This angle has a potential arising from instantons which causes $(a / f)$ to select the vacuum $\bar{\theta}=0$. The axion will, in general, have additional derivative couplings to matter, such as a modeldependent coupling to photons of the form $(a / f) F \tilde{F}$ $[13,14]$. The mass of the axion $m_{a}$ satisfies $m_{a} \approx$ $m_{\pi} f_{\pi} / f$, where $m_{\pi} \approx 140 \mathrm{MeV}$ and $f_{\pi} \approx 93 \mathrm{MeV}$ are the pion mass and decay constant, respectively.

The axion decay constant is bounded from below by collider experiments and astrophysical arguments. The latter are the more stringent: If $f$ is too small, the coupling to ordinary matter is large enough to allow rapid axion production in red giants and supernovae, leading to an unacceptably large cooling rate. This yields the lower bound $10^{9} \mathrm{GeV} \lesssim f$ [15]. Large $f$ leads to copious production of cold, degenerate axions in the early Universe [16-18], so that $f \geq 10^{12} \mathrm{GeV}$ leads to unacceptably large $\Omega_{\mathrm{dm}}$, where $\Omega_{\mathrm{dm}}$ is the fraction of dark matter in the Universe today, measured to be 0.21 to within $4 \%$ [19]. Therefore, the axion decay constant is conventionally assumed to lie in the window $10^{9} \mathrm{GeV} \lesssim f \leqq 10^{12} \mathrm{GeV}$. At the upper end of this bound, axions are a viable dark matter candidate, and experimental attempts to detect their presence are in progress [20].
In this Letter, we will present an exotic cosmological scenario for axions, and so we first summarize the conventional picture. The PQ symmetry is assumed to break spontaneously at a temperature $T \sim f$ well above the QCD scale where the instanton induced axion potential turns on. Following this phase transition, $(a / f)$ equals some random angle $\theta_{i}$ until $T \sim 1 \mathrm{GeV}$, below which the axion potential develops rapidly. The axion field begins to oscillate at temperature $T_{i} \sim 1 \mathrm{GeV}$ when the axion mass comes within the horizon, $m_{a}\left(T_{i}\right) \approx H\left(T_{i}\right)$, where $H\left(T_{i}\right)$ is the Hubble parameter at temperature $T_{i}$, which is only weakly dependent on $f$. The coherent oscillation may be thought of as a gas of degenerate nonrelativistic axions with the number density of axions per comoving volume equal to $n_{a}=\theta_{i}^{2} H\left(T_{i}\right) f^{2}$. This quantity remains constant, since annihilation rates are negligible. As a result, the subsequent axion energy density at temperature $T$ is given by $\rho_{a}=m_{a} n_{a}\left(R\left(T_{i}\right) / R(T)\right)^{3}$, where $R(T)$ is the RobertsonWalker scale factor at temperature $T$. The upper bound on $f$ follows from observational limits on $\Omega_{\mathrm{dm}}$, since $\rho_{a}$ varies almost linearly with $f$, assuming that $\theta_{i}=O(1)$.

There have been prior attempts to evade the cosmological bound, motivated in part by the fact that, in string theories, axions with $f=\sqrt{2} \alpha_{U} M_{p} \approx 10^{16} \mathrm{GeV}$, where $\alpha_{U}$ is the unified value of the fine structure constant, are generic. A trivial way to harmlessly incorporate an axion $A$ with decay constant $F>10^{12} \mathrm{GeV}$ is to introduce a second pseudoscalar $a$ so that the Lagrangian contains the term $(A / F+a / f) G \tilde{G}$, in which case the spectrum can consist of an innocuous massless boson and a conventional axion with decay constant $f$. Another resolution is to assume inflation and an ensemble of initial angles $\theta_{i}$ to choose from, invoking the anthropic principle to justify a value in our Universe of $\theta_{i} \approx 0$ [21]; recently, it was pointed out that such a scenario could be constrained by the Planck polarimetry experiment [22]. Alternatively, in Ref. [23] the axion is coupled to light quarks in such a way as to allow for exotic axion cosmology below the QCD scale.

In this Letter, we explore unconventional axion cosmology without invoking the anthropic principle and without convoluting the PQ mechanism with flavor physics; in common with Refs. [23,24], our scenario involves late evolution of the PQ symmetry breaking order parameter, 
although it differs significantly in realization and phenomenology. The conventional cosmological bound on $f$ results because the PQ symmetry spontaneously breaks at a temperature well before the QCD scale. However, if $f$ only evolves to a large vacuum expectation value (vev) after the QCD time, such bounds may be evaded. This could be accomplished with a sufficiently flat PQ potential, so that the radial mode only evolves out to a large vev once its mass enters the horizon.

The fine-tuning associated with such a flat potential can be avoided only with supersymmetry (SUSY); but even then, SUSY breaking will, in general, generate a curvature for the PQ potential which forces PQ breaking at or above the weak scale, a transition which is too early to evade the cosmological constraints. This problem can be avoided with the introduction of a new sector which couples only weakly to the standard model via the conventional PQ sector. We show that such a coupling may be weak enough to shield the potential in the new sector from SUSY breaking effects, while still significantly affecting axion phenomenology. Our model evades the problems of Ref. [25], where it was shown that, in supersymmetric theories, cosmological overproduction of saxions is typically more of a problem than an excess of axions.

The spectrum of our model includes (i) an axion far lighter than conventionally allowed with no significant cosmological abundance; (ii) an additional pseudoscalar which is heavier than would be an axion with comparable decay constant; (iii) a light dilatonlike scalar particle. In the model presented here, the latter two are dark matter candidates. In the next sections, we describe a model which realizes this scenario. While not particularly compelling as a description of nature, the model has been constructed to illustrate how model-dependent axion cosmology and detection can be, within a framework that successfully addresses the strong $C P$ problem.

A model. - Our starting point is to assume a viable supersymmetric theory which implements the conventional PQ mechanism. We assume that there exists a superfield $\phi_{1}$ which carries PQ charge and couples to colored fermions in a real representation of the gauge group; at a temperature well above the QCD scale, this field acquires a vev $\left\langle\phi_{1}\right\rangle=v_{1} / \sqrt{2}$, which lies within the conventional window $10^{9} \mathrm{GeV} \lesssim v_{1} \lesssim 10^{12} \mathrm{GeV}$. With this vev, the heavy colored fermions coupled to $\phi_{1}$ develop a mass $M_{Q}=g v_{1}$. It is important for our modification of the theory that the saxion be light (to be specified below) so that it not communicate large SUSY breaking to a new sector we will be adding. A light saxion is expected in any theory of low energy SUSY breaking; it could also occur in gravity-mediated SUSY breaking models, so long as the PQ sector is sequestered from the SUSY breaking. An excess of saxion energy can be avoided in such models either by having relatively late inflation (below the PQ breaking scale but well above the QCD time) or by having the minimum of the saxion potential be at the same point as preferred by finite temperature effects prior to an epoch of higher scale inflation.

To this theory, we now introduce the superpotential

$$
\tilde{W}=\sqrt{2} \lambda A\left(h \phi_{1} \phi_{2}-\phi_{0}^{2}\right)+\frac{1}{\sqrt{2}} \mu^{2} B\left(2\left(\phi_{0} / v_{0}\right)^{2}-1\right),
$$

where $\phi_{2}$ carries opposite PQ charge from $\phi_{1}$, while $A, B$, and $\phi_{0}$ are PQ-invariant fields. The four parameters $v_{0}^{2}, \mu^{2}$, $h$, and $\lambda$ may all be taken to be real by redefinition of the phases of $A, B, \phi_{0}$, and $\phi_{2}$. The part of the scalar potential relevant to us is

$\tilde{V}\left(\phi_{1}, \phi_{2}, \phi_{0}\right)=2 \lambda^{2}\left|h \phi_{1} \phi_{2}-\phi_{0}^{2}\right|^{2}+\frac{\mu^{4}}{2}\left|2\left(\phi_{0} / v_{0}\right)^{2}-1\right|^{2}$.

The first term in $\tilde{V}$ exhibits a flat direction in $\phi_{2}$ and $\phi_{0}$, which is lifted slightly by the second term in $\tilde{V}$, with $\lambda \ll$ 1 and $\mu \ll \Lambda_{\mathrm{QCD}}$. The smallness of the couplings leaves $\sqrt{2}\left\langle\phi_{1}\right\rangle=v_{1}$ unaffected, and the minimum of the almost flat direction is at $\sqrt{2}\left\langle\phi_{0}\right\rangle=v_{0} \gg v_{1}$, and $\sqrt{2}\left\langle\phi_{2}\right\rangle=$ $v_{2}=v_{0}^{2} /\left(h v_{1}\right) \gg v_{0}$.

We assume that following inflation and reheating the Universe sits away from the minimum of the potential with $\sqrt{2}\left\langle\phi_{1}\right\rangle=v_{1}$, and $\langle A\rangle=\langle B\rangle=\left\langle\phi_{0}\right\rangle=\left\langle\phi_{2}\right\rangle=0$, the latter being determined by high temperature effects due to interactions with unspecified heavy fields prior to inflation. This field configuration persists down to a temperature $T_{0}<\Lambda_{\mathrm{QCD}}$, satisfying $\mu^{2} / v_{0} \approx H\left(T_{0}\right)$, when the curvature lifting the flat direction is sufficiently strong to overcome the Hubble friction. Then $\phi_{0}$ will roll out to its minimum at $\left\langle\phi_{0}\right\rangle=v_{0} \gg v_{1}$, causing $\phi_{2}$ to follow its flat direction out to $v_{2}=v_{0}^{2} /\left(h v_{1}\right) \gg v_{0}$. With $\mu \ll$ $\Lambda_{\mathrm{QCD}}$, this phase transition will occur at $T_{0}<\Lambda_{\mathrm{QCD}}$, and, as we discuss further below, the dark matter produced in this transition can be made acceptable.

To understand how the pseudoscalars behave in this model, we expand around the minimum of the potential, writing $\phi_{i}=\frac{1}{\sqrt{2}}\left(v_{i}+\sigma_{i}\right) e^{i \pi_{i} / v_{1}}$, with $i=0,1,2$. The fields $\sigma_{i}$ and $\pi_{i}$ are the scalar and pseudoscalar excitations, respectively. After adding to $\tilde{V}$ the QCD contribution to the $\pi_{1}$ potential, the complete pseudoscalar potential is

$$
\begin{aligned}
V_{\pi}= & -m_{\pi}^{2} f_{\pi}^{2} \cos \frac{\pi_{1}}{v_{1}}-\lambda^{2} v_{0}^{4} \cos \left(\frac{\pi_{1}}{v_{1}}+\frac{\pi_{2}}{v_{2}}-\frac{2 \pi_{0}}{v_{0}}\right) \\
& -\mu^{4} \cos \left(\frac{2 \pi_{0}}{v_{0}}\right)
\end{aligned}
$$

[up to an overall $O(1)$ factor [13] in front of the first term which does not concern us here]. Since $\pi_{1}$ is the only field that couples to ordinary matter, to understand phenomenology we must decompose $\pi_{1}$ into mass eigenstates $a_{1,2,3}$. We diagonalize the mass matrix to leading nonzero order in $\delta=v_{1} / v_{0}$ and $\epsilon=\mu^{4} /\left(\lambda^{2} v_{0}^{4}\right)$ and to all orders in $x=$ $\lambda^{2} v_{0}^{4} /\left(m_{\pi}^{2} f_{\pi}^{2}\right)$, finding the masses and decay constants 


$$
\begin{gathered}
m_{a_{1}}^{2}=\frac{\lambda^{2} v_{0}^{4}}{v_{1}^{2}}\left(\frac{x}{1+x}\right), \quad f_{1}=v_{1}, \\
m_{a_{2}}^{2}=\frac{m_{\pi}^{2} f_{\pi}^{2}}{v_{0}^{2}}\left(\frac{x}{1+x}\right), \quad f_{2}=v_{0}\left(\frac{1+x}{2 x}\right), \\
m_{a_{3}}^{2}=\frac{\mu^{4}}{v_{0}^{2}},
\end{gathered}
$$

where the decay constants are defined by

$$
\mathcal{L}_{\mathrm{QCD}}=\frac{\pi_{1}}{v_{1}} G \tilde{G}=\left(\frac{a_{1}}{f_{1}}+\frac{a_{2}}{f_{2}}\right) G \tilde{G} .
$$

In these formulas, we have neglected terms of order $v_{0} / v_{2}$; the $a_{3}$ pseudoscalar decouples from the standard model to the order we work. Note that $x$ may be $\gg 1$.

This model evades the conventional constraints on the axion decay constant as the axion $a_{2}$ has a decay constant far in excess of $10^{12} \mathrm{GeV}$-it is potentially an axion with string scale PQ constant $f_{2} \approx 10^{16} \mathrm{GeV}$-yet is cosmologically unpopulated. The pseudoscalar $a_{1}$ also couples to $G \tilde{G}$, but, unlike an axion, its mass does not vanish in the limit that its coupling to $G \tilde{G}$ vanishes; its mass may, in fact, be far in excess of that of a conventional axion. The energy originally in $\pi_{1}$ is primarily transferred into the $a_{1}$ field, making it a good dark matter candidate; the light $a_{2}$ axion receives only a small fraction of that energy, suppressed by $\left(v_{1} / v_{0}\right)^{2} \ll 1$. The axionlike pseudoscalar $a_{1}$ may be detectable by experiments searching for cosmologically abundant axions; however, its mass may lie outside the window in which these experiments are currently searching.

Constraints. - At temperatures below the QCD scale but above the secondary transition, the Universe has a background density of cold $\pi_{1}$ pseudoscalars which behave like a conventional axion with decay constant $v_{1}$. Subsequently, the $\phi_{0}$ field rolls out to its minimum $\left\langle\phi_{0}\right\rangle=v_{0}$ at the temperature $T_{0}$ where $H\left(T_{0}\right) \sim \mu^{2} / v_{0}$. At this point (i) the energy in $\pi_{1}$ pseudoscalars is redistributed among the $a_{1}$ and $a_{2}$ mass eigenstates following Eq. (4), and (ii) an energy density $\mu^{4}$ is released and is mostly transferred into $\sigma_{0}$ (radial) oscillations of the $\phi_{0}$ field. There are two cosmological constraints on the late transition. The first is that the combined energy density in the exotic $a_{1}$ pseudoscalar and $\sigma_{0}$ scalar not exceed the observed dark matter density today. A second constraint is that the phase transition occur after the QCD time but before today (or well before matter-radiation equality if either $a_{1}$ or $\sigma_{0}$ contribute an appreciable fraction of the dark matter). In addition, there is the astrophysical constraint that, to prevent copious production of any of the light pseudoscalars in supernovae, we require $f_{1}, f_{2} \geqslant 10^{9} \mathrm{GeV}$. We now discuss these constraints in detail and map out the corresponding parameter space.

In order to ensure no excess of dark matter, we must limit the energy in $\pi_{1}$ by requiring $f_{1} \lesssim 10^{12} \mathrm{GeV}$. We also require that the energy $\rho_{0}$ in the form of $\sigma_{0}$ oscillations produced at the secondary transition not dominate the Universe at the epoch of matter-radiation equality, $T_{\mathrm{eq}} \approx 1 \mathrm{eV}$ :

$$
\rho_{0}\left(T_{\mathrm{eq}}\right) \approx \mu^{4}\left(\frac{T_{\mathrm{eq}}}{T_{0}}\right)^{3} \lesssim T_{\mathrm{eq}}^{4}, \quad H\left(T_{0}\right) \approx \frac{\mu^{2}}{v_{0}} .
$$

A complication arises from the fact that energy in the early $\pi_{1}$ oscillations is transferred primarily into $a_{1}$ oscillations, while $a_{1}$ becomes heavier than $\pi_{1}$ by a factor of $\sqrt{1+x}$ as $\phi_{0}$ rolls from $\phi_{0}=0$ to $\phi_{0}=v_{0}$. This increase of energy must come from the energy released during the secondary transition; it can be represented by a contribution to the potential for $\phi_{0}$ due to the $\phi_{0}$-dependent energy density of the $a_{1}$ pseudoscalar condensate, of the form

$$
\rho_{1}\left(\phi_{0}, T\right)=n_{\pi_{1}}(T) m_{\pi_{1}} \sqrt{1+x\left(\phi_{0}\right)},
$$

where $T_{i} \sim 1 \mathrm{GeV}$ is the temperature when $\pi_{1}$ begins to oscillate, $n_{\pi_{1}}(T)=\left(\theta_{i}^{2} H_{i} f_{1}^{2}\right)\left(T / T_{i}\right)^{3}$ is the number density of $\pi_{1}$ bosons in the temperature range $\Lambda_{\mathrm{QCD}} \geq T \geq T_{0}$, $m_{\pi_{1}}=\left(m_{\pi} f_{\pi} / f_{1}\right)$ is the $\pi_{1}$ mass before $\phi_{0}$ rolls out, and $x\left(\phi_{0}\right)=\left(\phi_{0} / v_{0}\right)^{4} x$ controls how the $\pi_{1}$ mass becomes the $a_{1}$ mass as $\phi_{0}$ increases. If $\rho_{1}\left(v_{0}, T_{0}\right)>\mu^{4}$, this potential delays the secondary transition, so that $\phi_{0}$ only gains its vev at some lower temperature $T=T_{0}^{\prime}$ satisfying $\rho_{1}\left(v_{0}, T_{0}^{\prime}\right) \approx \rho_{0}\left(T_{0}^{\prime}\right) \approx \mu^{4}$. After this delayed transition, the energy density in both $\phi_{0}$ and $a_{1}$ oscillations remains comparable, diluting $\propto T^{-3}$. After a little algebra, one finds that Eq. (6) still holds in this case but is augmented by the constraint that there not be too much energy in the cosmological $a_{1}$ abundance,

$$
T_{\mathrm{eq}}^{4} \gtrsim\left(\frac{T_{\mathrm{eq}}}{T_{0}}\right)^{3} n_{\pi_{1}}\left(T_{0}\right) m_{a_{1}}
$$

with $m_{a_{1}}$ defined in Eq. (4) and $T_{0} \approx \sqrt{M_{p} \mu^{2} / v_{0}}$ from Eq. (6). If we now assume $\rho_{1}+\rho_{0}=\rho_{\mathrm{DM}}$, that is, that $a_{1}$ and $\sigma_{0}$ together compose the dark matter, we can use Eq. (8) to compute allowed masses and couplings, $f_{1}$, $m_{a_{1}}$, for the observable dark matter candidate $a_{1}$. The result is shown in Fig. 1; while the $a_{1}$ pseudoscalar couples to ordinary matter as a conventional axion would, its mass generally exceeds that of an axion, a result of its coupling with the sector that generates the exotic axion $a_{2}$.

Finally, we obtain a constraint from requiring $\phi_{0}$ to roll after the QCD phase transition, but somewhat before matter-radiation equilibrium if either $a_{1}$ or $\sigma_{0}$ are to be the dark matter:

$$
T_{\mathrm{eq}}\left(\frac{v_{0}}{M_{p l}}\right)^{1 / 2} \lesssim \mu \lesssim T_{\mathrm{QCD}}\left(\frac{v_{0}}{M_{p l}}\right)^{1 / 2} .
$$

If the origin of dark matter lies elsewhere, then $T_{\mathrm{eq}}$ in the above equation is replaced by today's temperature.

The model as it stands possesses an exact discrete symmetry $\phi_{0} \rightarrow-\phi_{0}$ which is spontaneously broken and leads to domain walls. This can be avoided by breaking the symmetry explicitly, either with a small linear term in the low energy superpotential Eq. (1) or in the high energy 


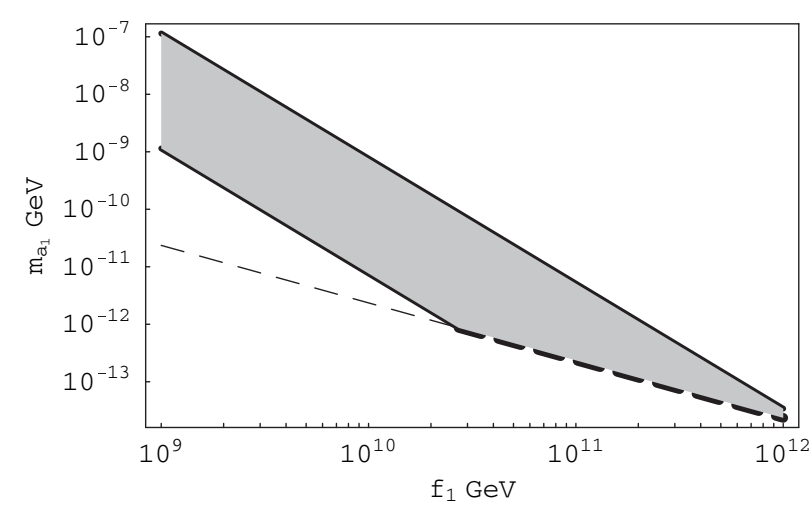

FIG. 1. The mass of the exotic pseudoscalar $a_{1}$ vs its decay constant $f_{1}$. Two solid parallel lines give $m_{a_{1}}\left(f_{1}\right)$, assuming $a_{1}$ comprises half the dark matter for initial misalignment angle $\theta_{i}=0.1$ (upper line) and $\theta_{i}=1$ (lower line). The dashed line is the pseudoscalar mass in standard PQ models, with the heavy dashed line giving the region where conventional axions could be the dark matter.

interactions such that $\left\langle\phi_{0}\right\rangle \neq 0$ (but $\lesssim v_{1}$ ) after postinflationary reheating.

Aside from cosmological constraints, there is also a naturalness constraint. Having a flat potential is critical for the late phase transition, so we require that SUSY breaking terms induced by the interactions between $\phi_{1}$, $\phi_{2}$, and $\phi_{0}$ do not lift the flat direction too much. The most important term is the soft mass generated for $\phi_{2}$ from the SUSY breaking $\phi_{1}$ mass, for which we require

$$
\Delta m_{2}^{2} f_{2}^{2} \approx \frac{m_{a_{1}}^{2} m_{\phi_{1}}^{2}}{16 \pi^{2}} \lesssim \mu^{4}
$$

This imposes a significant new constraint on $\mu$. In addition, we are assuming this sector is sequestered from gravitymediated SUSY breaking [26].

The combination of constraints Eqs. (6) and (8)-(10) yields a parameter space too large to explore in detail here. We give here instead two representative sets of values in Table I satisfying the constraints.

Phenomenology. - Conventional axion models have fairly circumscribed phenomenology; if one assumes that the dark matter consists of a conventional axion, then the mass and coupling of the axion are related in a direct way, and both lie in a fairly model independent range about $m_{a} \sim 10^{-5} \mathrm{eV}, f_{a} \sim 10^{12} \mathrm{GeV}$ determined by the initial axion misalignment $\theta_{i}$. If the axion is not the dark matter, its mass can be heavier.

In contrast, we have shown how these simple relations can be greatly modified in a theory with a late phase transition below the QCD scale, as shown in Table I. Dark matter in this theory can be comprised of roughly equal parts of remnant pseudoscalar and scalar particles.

It will be interesting to investigate the consequences of such late phase transitions for structure formation.
TABLE I. Two sets of parameters (I, II) allowed by the constraints Eqs. (6) and (8)-(10). Set I gives parameters for a prompt transition at temperature $T_{0}$; set II gives parameters for a delayed transition at $T_{0}^{\prime}$. All parameters are in $\mathrm{GeV}$ except the pseudoscalar masses, which are in $\mathrm{eV}$.

\begin{tabular}{lccccccc}
\hline \hline & $m_{a_{1}}$ & $f_{1}$ & $m_{a_{2}}$ & $f_{2}$ & $x$ & $\mu$ & $T_{0}\left(T_{0}^{\prime}\right)$ \\
\hline I & $10^{-2}$ & $10^{10}$ & $10^{-5}$ & $10^{15}$ & 10 & $10^{-3}$ & $10^{-2}$ \\
II & 1 & $10^{10}$ & $10^{-9}$ & $10^{16}$ & $10^{3}$ & $10^{-6}$ & $10^{-6}$ \\
\hline \hline
\end{tabular}

We are grateful for numerous conversations about this work with C. Hogan and A. Nelson. This work was supported by DOE Grant No. DE-FGO3-00ER41132.

*Electronic address: dbkaplan@phys.washington.edu †Electronic address: zurkat@u.washington.edu

[1] A. E. Nelson, Phys. Lett. 136B, 387 (1984).

[2] S. M. Barr, Phys. Rev. D 30, 1805 (1984).

[3] R. D. Peccei and H. R. Quinn, Phys. Rev. Lett. 38, 1440 (1977).

[4] R. D. Peccei and H.R. Quinn, Phys. Rev. D 16, 1791 (1977).

[5] R. N. Mohapatra and G. Senjanovic, Phys. Lett. 79B, 283 (1978).

[6] K. S. Babu, B. Dutta, and R. N. Mohapatra, Phys. Rev. D 65, 016005 (2002).

[7] S. Weinberg, Phys. Rev. Lett. 40, 223 (1978).

[8] F. Wilczek, Phys. Rev. Lett. 40, 279 (1978).

[9] J. E. Kim, Phys. Rev. Lett. 43, 103 (1979).

[10] M. A. Shifman, A. I. Vainshtein, and V. I. Zakharov, Nucl. Phys. B166, 493 (1980).

[11] A. R. Zhitnitsky, Sov. J. Nucl. Phys. 31, 260 (1980).

[12] M. Dine, W. Fischler, and M. Srednicki, Phys. Lett. 104B, 199 (1981).

[13] D. B. Kaplan, Nucl. Phys. B260, 215 (1985).

[14] M. Srednicki, Nucl. Phys. B260, 689 (1985).

[15] G. G. Raffelt, Stars as Laboratories for Fundamental Physics (Chicago University Press, Chicago, 1996).

[16] J. Preskill, M. B. Wise, and F. Wilczek, Phys. Lett. 120B, 127 (1983).

[17] L. F. Abbott and P. Sikivie, Phys. Lett. 120B, 133 (1983).

[18] M. Dine and W. Fischler, Phys. Lett. 120B, 137 (1983).

[19] S. Eidelman et al. (Particle Data Group), Phys. Lett. B 592, 1 (2004); URL http://pdg.lbl.gov.

[20] L. J. Rosenberg, in Proceedings of the 32nd SLAC Summer Institute on Particle Physics: Nature's Greatest Puzzles, Menlo Park, CA, 2004, eConf C040802, MOT002 (2004).

[21] A. D. Linde, Phys. Lett. B 201, 437 (1988).

[22] P. Fox, A. Pierce, and S. Thomas, hep-th/0409059.

[23] S. M. Barr and B. Kyae, Phys. Rev. D 71, 055006 (2005).

[24] S. M. Barr, Phys. Lett. B 454, 92 (1999).

[25] T. Banks, M. Dine, and M. Graesser, Phys. Rev. D 68, 075011 (2003).

[26] L. Randall and R. Sundrum, Nucl. Phys. B557, 79 (1999). 